\title{
Apego al Lugar e Identidad de Lugar en Barrios Patrimoniales
}

\section{Place Attachment and Place Identity in Heritage Neighborhoods}

\author{
Héctor Berroeta ${ }^{1} \triangle \mathrm{ORCID}$, Héctor Marcelo Rodríguez Mancilla ${ }^{2} \mathrm{ORCID}$, \\ Marcos Zumárraga-Espinosa ${ }^{3} \mathrm{ORCID}$ \\ ${ }^{1}$ Universidad de Valparaíso, Chile. \\ 2 Universidad de Playa Ancha, Chile. \\ ${ }^{3}$ Universidad Politécnica Salesiana, Ecuador.
}

Fecha correspondencia:

Recibido: junio 6 de 2020.

Aceptado: abril 6 de 2021.

Forma de citar:

Berroeta, H., Rodríguez Mancilla, H.M, \& Zumárraga-Espinosa, M. (2021).

Apego al Lugar e Identidad de Lugar en Barrios Patrimoniales. Rev. CES

Psico, 14(1), 85-99.

Open access

(c) Copyright

Licencia creative commons

Etica de publicaciones

Revisión por pares

Gestión por Open Journal System

DOI: http://dx.doi.org/10.21615/

cesp.14.1.7

ISSN: 2011-3080

Sobre los autores:

1. Doctor por la Universidad de Barcelona. Profesor titular en la Universidad de Valparaíso, Chile. Director alterno del Centro de Investigaciones en Vulnerabilidades

Comparte

f日G.

\section{Resumen}

La política internacional de protección del patrimonio mundial de la UNESCO se integró en las agendas de desarrollo nacional y local de los países de América Latina, gestionando proyectos de mejoramiento social y urbano en barrios históricos de las ciudades. Los efectos sociales, ambientales y económicos de estos procesos de patrimonialización de los lugares no han sido suficientemente analizados. En este sentido, y asumiendo una perspectiva psicoambiental, esta investigación describe y compara los índices de Apego al Lugar e Identidad de Lugar en habitantes de tres barrios de la ciudad de Valparaíso ( $n=544$ ) (Chile) y dos en la ciudad de Quito ( $n=209)$ (Ecuador), que se encuentran ubicados en zonas catalogadas como Patrimonio de la Humanidad. A través de la aplicación de pruebas $t$ de Student de comparación de medias y $d$ de Cohen de valoración del tamaño del efecto, se observó que las personas que cuentan con mayor tiempo de residencia, que habitan barrios patrimoniales consolidados (donde las dimensiones socio-urbanas del entorno se encuentran mayormente intervenidas y desarrolladas) y que son propietarias de sus viviendas presentan puntajes más altos de Apego al Lugar e Identidad de Lugar. Se observó un nivel moderado de Apego de Lugar e Identidad de Lugar en los habitantes de los barrios patrimoniales de las ciudades de Valparaíso y Quito.

Palabras clave: Apego al Lugar, Identidad de Lugar, Barrios Patrimoniales, Renovación Urbana, Sentido de Comunidad, Participación Ciudadana.

\section{Abstract}

UNESCO's international policy for the protection of world heritage was integrated into the national and local development agendas of Latin American countries, managing social and urban improvement projects in historic city districts. The social, environmental and economic effects of these processes of heritage sites have not been sufficiently analyzed. In this sense and assuming a psycho-environmental perspective, this research describes and compares the indices of place attachment and place identity in 3 neighborhoods in the city of Valparaiso $(n=544)$ and 2 in the city of Quito $(n=209)$, which are located in areas classified as World 
e Informalidades territoriales CINVIT. Investigador Responsable proyecto Fondecyt 1181429.

2. Doctor en Planificación Urbana y Regional. Psicólogo. Profesor Asociado Departamento de Psicología, Universidad de Playa Ancha, Chile. Investigador del Observatorio de Participación Social y Territorio, Universidad de Playa Ancha, Chile.

3. Magíster en Gestión Pública. Economista. Docente investigador de la Universidad Politécnica Salesiana, Ecuador (UPS). Miembro del Grupo de Investigaciones Psicosociales (GIPS-UPS) y del Grupo de Innovación Educativa (GIE-UPS) de Orientación Vocacional y Profesional.
Heritage. Through the application of Student $t$ tests for comparing means and Cohen $d$ tests for assessing the size of the effect, it was observed that people who have resided for longer periods of time, who live in consolidated heritage neighborhoods and who own their own homes, show higher scores in terms of place attachment and place identity. A moderate level of Place Attachment and Place Identity was observed in Valparaíso and Quito.

Keywords: Place Attachment, Place Identity, Heritage Sites, Urban Renewal, Sense of Community, Citizen Participation.

\section{Introducción}

Con la Organización de las Naciones Unidas por la Educación la Ciencia y la Cultura (UNESCO) y la convención sobre la protección del patrimonio mundial, cultural y natural de 1972, se inicia un proceso de mundialización de los valores asociados a las prácticas patrimoniales. Esto impulsó la creación de un amplio sistema de cooperación internacional para definir lugares en las ciudades con alto valor simbólico, arquitectónico, natural e histórico, dignos de ser conservados. Son patrimonio universal los monumentos, conjuntos edificados, yacimientos arqueológicos o conjuntos que presentan un valor universal excepcional desde el punto de vista de la historia del arte o de la ciencia (Choay, 1992).

Esta política internacional se fue integrando progresivamente en las agendas de desarrollo nacional y local de los países de América Latina, de modo que se gestionaron proyectos de mejoramiento social y urbano. Esto propició un amplio debate conceptual y operativo en torno al proceso de patrimonialización de los lugares y sus efectos sociales, ambientales, económicos, educativos y culturales proyectados en las agendas de desarrollo (Galaz-Mandakovic, 2019; Leiva \& Díaz, 2020). En términos generales, el debate ha transitado desde una concepción esencialista y monumentalista del patrimonio, cuyo significado es estable y unitario, hacia una visión del patrimonio como construcción social, es decir, como elemento dinámico, histórico, simbólico y situado (Berroeta, 2009; Gutman, 2001; Lacarrieu, 2004).

En este marco se han venido desarrollando, en mayor medida en América Latina, estudios que analizan: a) los procesos de gentrificación que remiten al recambio de población de bajos ingresos económicos por población de mayores ingresos, producto de transformaciones del espacio urbano en sectores deteriorados que aumentan los precios de arriendo y del costo de vida (Casellas \& Vergara-Constela, 2016; Cuenya, 2011; Guevara, 2011; Suárez, 2010); b) los efectos excluyentes que generan este tipo de políticas (Andrade, 2018; Cabrera, 2008; Hanley, 2008; Rodríguez, 2015); c) la invisibilización de las culturas subalternas y su folklorización (Lacarrieu \& Laborde, 2018; Kingman \& Prats, 2008), a partir de la institucionalización de la cultura (Kingman, 2004); d) la eficacia y efectividad de las políticas patrimoniales (López, 2014); y e) las tensiones y disputas por el espacio urbano que se dan por la revalorización económica y turística de lugares y barrios deteriorados (Carrión, 2005; Galaz-Mandakovic, 2019; Hanley, 2008; Salcedo, 2007).

Sin embargo, un aspecto que no ha sido explorado de manera específica en Latinoamérica, y que se vincula directamente con los efectos de los procesos de patrimonialización a escala barrial, es la relación simbólica y afectiva que se da entre las personas y los entornos patrimonializados. Investigaciones asociadas al turismo (Loureiro \& Sarmento, 2018; Su \& Wall, 2010; Woosnam et al., 2018) dan cuenta de la importancia que tiene para la gestión patrimonial, considerar los vínculos psi- 
Para la Psicología Ambiental, el Apego al Lugar y la Identidad de Lugar son los principales conceptos que explican la relación entre las personas y los entornos construidos. coambientales de residentes y turistas. En efecto, se ha mostrado que la motivación cultural en términos de información y conocimiento de sitios patrimoniales influye positivamente en el Apego al Lugar (Latiff et al., 2020), el cual incide en el desarrollo de comportamientos sostenibles (Marasco, Buonincontri1, \& Ramkissoon, 2018).

Para la Psicología Ambiental, el Apego al Lugar y la Identidad de Lugar son los principales conceptos que explican la relación entre las personas y los entornos construidos. Estos vínculos han sido ampliamente investigados (Brown, Perkins, \& Brown 2003; Brown \& Raymond, 2007; Hernández, Hidalgo, Salazar-Laplace, \& Hess, 2007; Manzo, 2003) y en distintos ámbitos espaciales, como el hogar (Cuba \& Hummon, 1993; Hidalgo \& Hernández, 2001; Tuan, 1974), las ciudades (Boniato, Aiello, Perugini, Bonnes, \& Ercolano, 1999; Vidal, Valera \& Peró, 2010), y los barrios, donde las investigaciones se han desarrollado más profusamente (Brown et al., 2003; Cuba \& Hummon, 1993; Dallago et al., 2009; Hidalgo \& Hernández, 2001; Lewika, 2011). Si bien la literatura es copiosa, también es confusa respecto a la relación entre estos dos constructos. En ambos casos se distinguen aspectos vinculados al individuo y aspectos vinculados al entorno, incluso se ha planteado una identidad de lugar de las personas y una identidad del lugar asociada a los espacios (Peng, Strijker, \& Wu, 2020).

El Apego al Lugar se refiere al vínculo emocional o afectivo que las personas establecen con los lugares (Lewicka, 2011; Scannell \& Gifford, 2010a, 2010b), expresado en la tendencia a mantener un grado de proximidad con los mismos (Giuliani, 2003; Hidalgo \& Hernández, 2001; Manzo, 2003; Pretty, Chipuer, \& Bramston, 2003; Williams, Patterson, Roggenbuck, \& Watson, 1992). Si bien, en el concepto de Apego al Lugar, las características físicas del entorno son consideradas un aspecto central en la conformación del vínculo persona entorno (Scannell \& Gifford, 2010a), la mayoría de las investigaciones han estado orientadas a identificar la fortaleza de este vínculo y las características de las personas que se apegan (Lewicka, 2011), dejando en segundo plano las características particulares de los entornos y su influencia en el Apego de Lugar.

Por su parte, la Identidad de Lugar se entiende como una subestructura cognitiva de la identidad personal conformada por las cogniciones sobre el mundo físico en el cual vive el individuo. Estas cogniciones representan recuerdos, ideas, sentimientos, actitudes, valores, preferencias, significados, y concepciones de conducta y experiencias relacionadas con la variedad y complejidad de los entornos físicos en los cuales los individuos se desenvuelven (Proshansky, Fabian, \& Kaminoff, 1983). Según Rijnks y Strijker (2013) la identificación de los individuos con un lugar puede reflejarse en las identidades que atribuyen al lugar, que posteriormente se incorporan a sus propias identidades.

En lo central, ambos conceptos parten del principio de que los entornos construidos transmiten a los individuos determinados significados que han sido socialmente elaborados y las personas, a su vez, interpretan estos significados y los transforman mediante un proceso de reelaboración que permite estabilizar los vínculos individuales con el lugar. Desde esta perspectiva, describir y analizar los vínculos que establecen con su entorno, quienes residen en barrios que están sometidos a diferentes procesos de transformación espacial por revalorización patrimonial, permite explorar el peso de la distintividad del lugar en la conformación del Apego al Lugar y la Identidad de Lugar, en tanto que, por sus características físicas, estos barrios son reconocidos por la UNESCO como distintivos de la ciudad en la que se encuentran. 
La presente investigación se propone describir, analizar y comparar los niveles de Apego al Lugar e Identidad de Lugar en tres barrios patrimoniales de la ciudad de Valparaíso, Chile y dos barrios patrimoniales de la ciudad de Quito, Ecuador.
En los barrios, el Apego al Lugar y la Identidad de Lugar se desarrollan como procesos psicosociales basados en actitudes hacia el ambiente local, caracterizado por interacciones sociales intensas y dinámicas (Brown, Brown, \& Perkins, 2004; Brown et al., 2003).

Estudios psicoambientales previos reportan diversas variables asociadas a las características de las relaciones sociales y a factores sociodemográficos como posibles predictores del Apego al Lugar y la Identidad de Lugar, tales como el nivel de los vínculos sociales en el barrio (Ahlbrandt, 1984; Ringel \& Finkelstein, 1991), el tiempo de residencia de los habitantes (Hernández, Hidalgo, Salazar-Laplace, \& Hess, 2007; Riger \& Lavrakas, 1981; Taylor, Gottfredson \& Brower, 1984,), el régimen de propiedad de la vivienda (Riger \& Lavrakas, 1981; Ringel \& Finkelstein, 1991; Taylor, Gottfredson, \& Brower, 1984), las expectativas de permanecer en el lugar actual (Riger \& Lavrakas, 1981) y la participación local (Cuba \& Hummon, 1993;Taylor, Gottfredson \& Brower, 1984), entre otras.

Dado lo anterior, la presente investigación se propone describir, analizar y comparar los niveles de Apego al Lugar e Identidad de Lugar en tres barrios patrimoniales de la ciudad de Valparaíso, Chile y dos barrios patrimoniales de la ciudad de Quito, Ecuador; buscando responder a las preguntas, ¿Qué niveles de Apego al Lugar e Identidad de lugar se observan en las personas que habitan barrios que han sido intervenidos por políticas dirigidas a valorizar zonas declaradas patrimoniales por la UNESCO? ¿Existen diferencias significativas entre los niveles de Apego al Lugar e Identidad de Lugar, y el tiempo de residencia, el tipo de barrio, la ciudad, la valoración de la condición patrimonial y el régimen de propiedad? La hipótesis de trabajo que se planteó sostiene que los niveles de Apego al Lugar e Identidad al Lugar son mayores cuando el tiempo de residencia en un lugar es más prolongado, en el caso de los barrios patrimoniales consolidados, cuando existe una valoración positiva del estatus patrimonial del barrio de residencia, y en personas que son propietarias de su vivienda.

Los casos de estudio seleccionados en la presente investigación corresponden a barrios patrimoniales de las ciudades de Valparaíso (Chile) y Quito (Ecuador), los cuales han sido objeto de procesos de intervención y mejoramiento social y urbano.

La ciudad de Valparaíso fue poblada en gran parte por inmigrantes europeos en la época del auge económico del puerto, quienes dejaron un importante legado arquitectónico y sociocultural, por el cual algunas zonas de la ciudad fueron nombradas Patrimonio de la Humanidad por la UNESCO en el año 2003. Una de estas zonas corresponde al casco histórico de los cerros Concepción, Santo Domingo y Cordillera, cada uno de los cuales han tenido un grado distinto de transformación. Mientras el barrio del cerro Concepción ha experimentado profundas transformaciones de su entorno y procesos de gentrificación con el apoyo de una fuerte inversión privada y pública orientada a la explotación turística, los barrios de los cerros Santo Domingo y Cordillera no se han transformado significativamente, y mantienen su población original, con altos índices de pobreza y precariedad urbana.

El centro histórico de la ciudad de Quito, a través del Plan de Reordenamiento Urbano de 1967, inspirado en la Carta de Venecia de 1964, se reconoce como hito material y símbolo de la fundación española de la ciudad. Este reconocimiento puso en el centro de las preocupaciones urbanas la necesidad de mejorar el espacio público y el 
conjunto de elementos patrimoniales, monumentales arquitectónicos y urbanísticos (Fondo de Salvamento del Patrimonio Cultural, 2009). El 8 de septiembre de 1978 la ciudad fue declarada Patrimonio Cultural de la Humanidad por la UNESCO. En los barrios "La Ronda" y "24 de Mayo", ubicados en el centro histórico de la ciudad, se iniciaron proyectos de renovación urbana en los años 2005 y 2006, respectivamente; con el propósito de promover el turismo y con inversión pública y privada. Ambos proyectos generaron cambios significativos en los entornos físicos, en su dinámica social se presentaron procesos de gentrificación, y se adelantaron acciones de control policial y municipal con el fin de expulsar de estos barrios tanto a personas en situación de calle, como a personas que trabajaban como comerciantes informales (López, 2014; Rodríguez, 2015).

\section{Método}

\section{Participantes}

El estudio se realizó en una muestra no probabilística por conveniencia constituida por habitantes de diferentes barrios de las ciudades de Quito-Ecuador y Valparaíso-Chile. En el caso de Quito se consideraron los barrios La Ronda y 24 de Mayo ( $n=209)$, y en Valparaíso se recopilaron datos de los barrios del Cerro Concepción, Santo Domingo y Cordillera $(n=544)$. La muestra global contó con 753 participantes. La submuestra de Quito se conformó por 117 mujeres y 92 hombres, con una media de edad de 41.3 años (DT = 15.9), y la de Valparaíso por 277 mujeres y 262 hombres, con una edad promedio registrada de 44.5 años $(D T=17.8)$

\section{Instrumentos}

Escala Global de Apego al Lugar (Hidalgo \& Hernández, 2001)

Mide la vinculación afectiva con el lugar y consta de cinco ítems con formato de respuesta tipo Likert (1=Nada a 6=Muchísimo), que se responden en relación con el barrio actual de residencia. Por ejemplo: "Me gusta vivir en este barrio". La estructura factorial de la escala original fue verificada a través de análisis factorial exploratorio. El análisis de confiabilidad se efectuó a través del coeficiente Alfa de Cronbach, y se obtuvo un valor estadísticamente satisfactorio $(\alpha=0.92)$. El índice de Apego al Lugar, correspondiente al puntaje agregado de la escala, se obtuvo promediando los cinco ítems que la conforman.

Escala Global de Identidad de Lugar (Vidal, Valera, \& Peró, 2010) Mide el grado de identificación con el lugar y consta de cinco ítems con formato de respuesta tipo Likert ( $1=$ Nada a $6=$ Muchísimo), que se responden en relación con el barrio actual de residencia. Por ejemplo: "Este barrio forma parte de mi identidad". La estructura factorial de la escala original fue verificada mediante análisis factorial exploratorio. La escala cuenta con un nivel aceptable de consistencia interna $(\alpha=0.86)$. El índice de Identidad de Lugar, correspondiente al puntaje agregado de la escala, se calculó promediando los cinco ítems que la integran.

\section{Cuestionario de recolección de variables}

Tiempo de residencia: El instrumento incluyó una pregunta por la cantidad de años de residencia en el lugar actual. Esta variable se categorizó en dos grupos: quienes han vivido por 5 años o menos en el lugar actual (34.8\%) y quienes han residido por un tiempo mayor a 5 años (65.2\%).

Tipo de barrio. Los barrios donde se efectuó la recolección de datos se agruparon en dos categorías: los barrios patrimoniales consolidados, tal es el caso de Cerro 
Pág 90

Una mirada general a estos resultados muestra que, considerando el rango teórico de los índices empleados [1 - 6], existe un nivel moderado de Apego al Lugar tanto en Valparaíso ( $M$ = 4.25) como en Quito ( $M=$ 4.04). La Identidad de Lugar, igualmente, exhibe un comportamiento moderado para Valparaíso $(M=4.06)$ y Quito $(M=3.86)$.
Concepción y La Ronda (44\%) y barrios patrimoniales no consolidados, que incluye a Santo Domingo, 24 de Mayo y Cordillera (56\%). Se entiende por consolidado aquel barrio donde las dimensiones socio-urbanas del entorno, como efecto de la nominación patrimonial y las políticas de renovación urbana, se encuentran mayormente intervenidas y desarrolladas.

Ciudad: El material empírico del presente estudio se obtuvo de barrios ubicados en las ciudades de Valparaíso-Chile (72.2\%) y Quito-Ecuador (27.8\%).

Valoración de la condición patrimonial: Se desarrolló un ítem con la finalidad de diferenciar entre los sujetos que evaluaban de forma positiva o negativa el estatus patrimonial de su lugar de residencia. La pregunta empleada fue: ¿Le gusta que su barrio sea patrimonio de la humanidad?

Régimen de propiedad: Los tipos de tenencia de la vivienda fueron recodificados en dos grupos: propietarios y quienes no son propietarios de la vivienda que habitan, esta última variable incluye alquiler u otras formas de residencia.

\section{Procedimiento}

Los instrumentos fueron aplicados en el lugar de residencia de los participantes por estudiantes de psicología que fueron debidamente capacitados. Los participantes firmaron previamente el respectivo consentimiento informado. La presente investigación fue aprobada por el comité de ética de la Universidad de Valparaíso, Chile.

\section{Análisis de datos}

Mediante pruebas $t$ de Student se compararon las medias de Apego e Identidad de Lugar en función del tiempo de residencia, tipo de barrio, ciudad, régimen de propiedad y valoración de la condición patrimonial. Para valorar la magnitud de las diferencias de medias encontradas (tamaño de efecto) se empleó la prueba $d$ de Cohen, considerando los siguientes criterios: valores menores a 0.20 indican una diferencia insignificante, de 0.21 a 0.50 una diferencia pequeña, de 0.51 a 0.80 diferencia mediana y valores mayores a 0.80 reflejan diferencias grandes (Cohen, 1992; Domínguez-Lara \& Campos-Uscanga, 2017). Para el análisis de datos se empleó el paquete estadístico SPSS 23.

\section{Resultados}

En la Tabla 1 se presenta un resumen de las medidas descriptivas de los índices de Apego al Lugar e Identidad de Lugar según las variables de agrupación de interés. Una mirada general a estos resultados muestra que, considerando el rango teórico de los índices empleados [1 - 6], existe un nivel moderado de Apego al Lugar tanto en Valparaíso $(M=4.25)$ como en Quito $(M=4.04)$. La Identidad de Lugar, igualmente, exhibe un comportamiento moderado para Valparaíso $(M=4.06)$ y Quito $(M$ = 3.86). En cuanto a los habitantes de los barrios patrimoniales consolidados, los datos muestrales revelan un grado moderado-alto de Apego al Lugar $(M=4.67)$ e Identidad de Lugar $(M=4.38)$, siendo superior al reportado por los habitantes de los barrios patrimoniales no consolidados.

Conjuntamente, en la Tabla 1 se reportan los resultados de las pruebas $t$ efectuadas, la estimación de tamaños de efecto y sus respectivos intervalos de confianza al 95\%. 
Pág 91

Tabla 1. Medias de Apego al Lugar e Identidad de Lugar en función de factores de interés, pruebas t y d de Cohen.

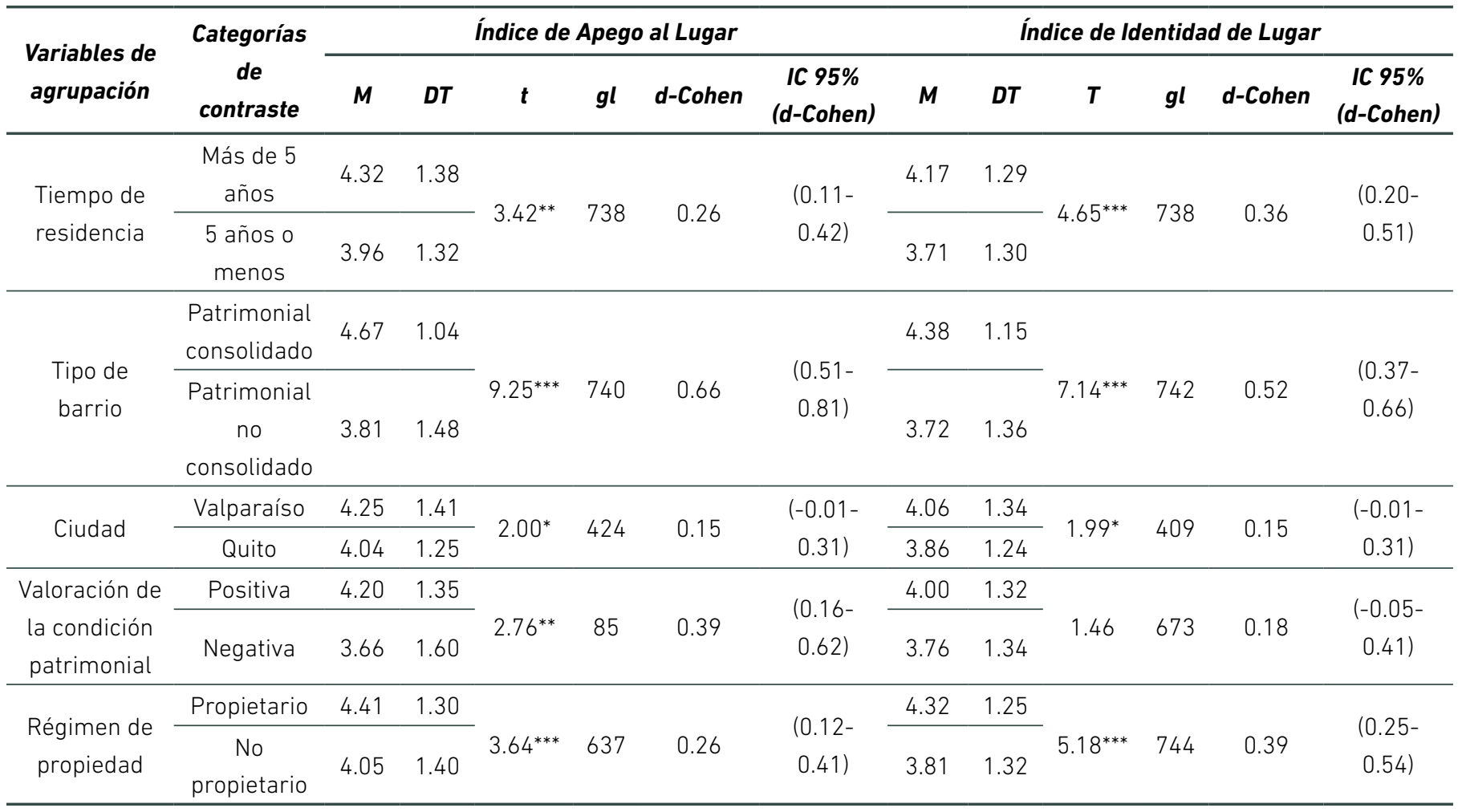

Nota: ${ }^{*} p<.05 ;{ }^{* *} p<.01 ;{ }^{* * *} p<.001$

Fuente: Datos recopilados por la Universidad de Valparaíso (Chile) y la Universidad Politécnica Salesiana (Ecuador). Elaboración propia.

Respecto al tiempo de residencia, quienes han vivido en el mismo lugar por un tiempo mayor a 5 años reportaron un nivel más alto de Apego al Lugar (t[738] $=3.42, p<$ 0.01 ) e Identidad de Lugar (t[738] $=4.65, \mathrm{p}<0.001)$, que quienes han residido por un tiempo menor o igual a 5 años (Ver Figura 1). Las medias de las variables Apego al Lugar ( $d=0.26$; IC95\% [0.11 - 0.42]) e Identidad de Lugar ( $d=0.36$; IC95\% [0.20 - 0.51]) difieren levemente.

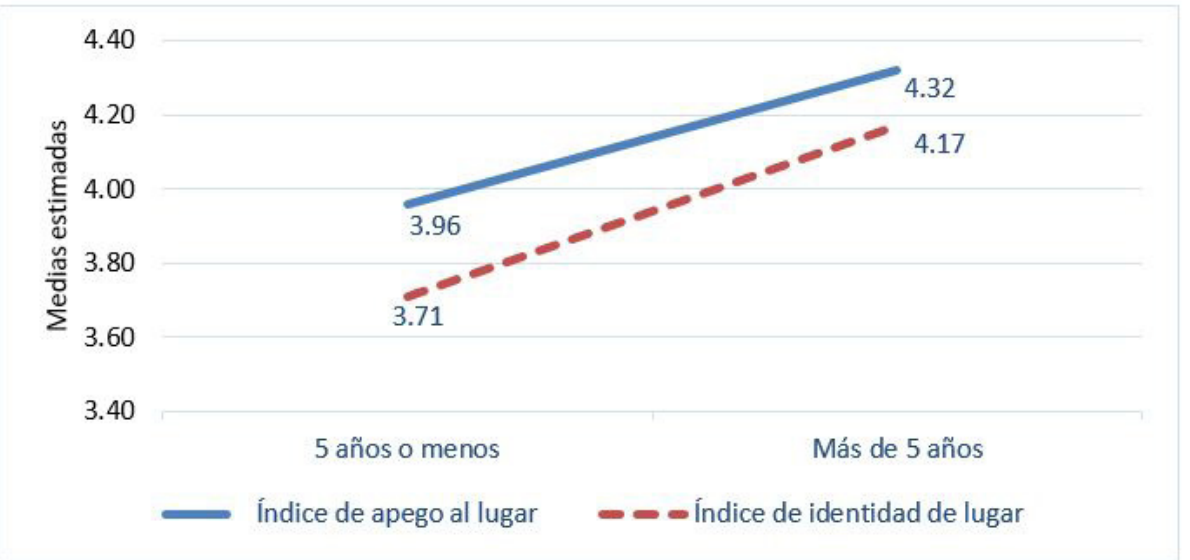

Figura 1. Medias de Apego al lugar e Identidad de lugar en función del tiempo de residencia. 
Se encontraron diferencias significativas en los puntajes de Apego al Lugar e Identidad de Lugar según el tipo de barrio y la valoración de la condición patrimonial (Ver Figura 2). Los barrios patrimoniales consolidados registraron una media más elevada de Apego al Lugar (t[740] = 9.25, $p<0.001)$ e Identidad de Lugar (t[742] = 7.14, $p<0.001)$ que los barrios patrimoniales no consolidados. Las estimaciones señalan tamaños de efecto moderados para Apego al Lugar ( $d=0.66$; IC95\% [0.51 - 0.81]) e Identidad de Lugar ( $d=0.52$; IC95\% [0.37 - 0.66]).

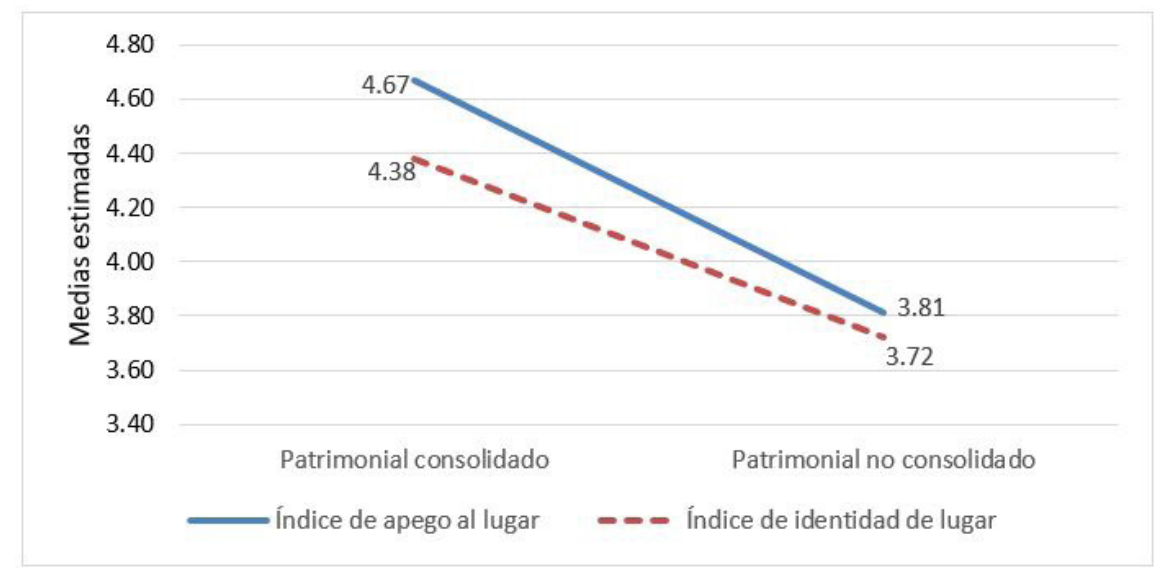

Figura 2. Medias de Apego al Lugar e Identidad de Lugar en función del tipo de barrio.

Fuente: Datos recopilados por la Universidad de Valparaíso (Chile) y la Universidad Politécnica Salesiana (Ecuador). Elaboración propia.

Al considerar la valoración de la condición patrimonial del lugar, sólo se encontraron diferencias significativas en términos de Apego al Lugar. Como se observa en la Figura 3, las personas que valoran positivamente la situación patrimonial del lugar que habitan, tienden a reportar mayor nivel de Apego al Lugar que quienes realizan una valoración negativa $(t[85]=2.76, p<0.01)$. El tamaño de efecto muestra una magnitud pequeña $(d=0.39$; IC95\% [0.16 - 0.62]). En contraste, los puntajes de Identidad de Lugar entre quienes realizaron valoraciones positivas y negativas no difirieron significativamente.

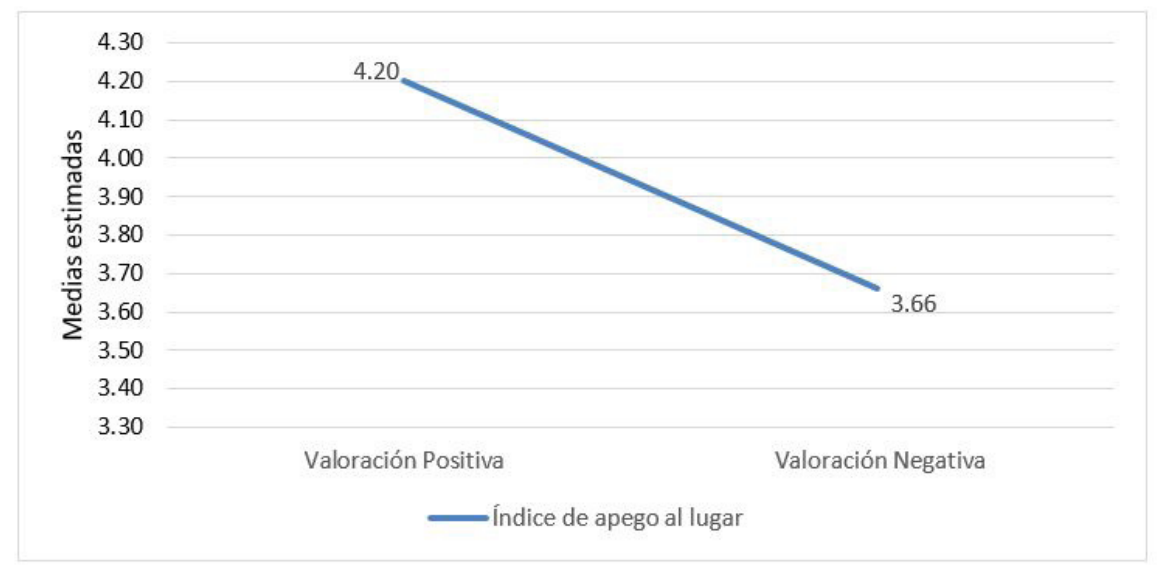

Figura 3. Medias de Apego al Lugar en función de la valoración subjetiva de la condición patrimonial del barrio 
Por último, las puntuaciones de Apego al Lugar e Identidad de Lugar variaron significativamente según el régimen de propiedad. Quienes son propietarios de su vivienda $(38,3 \%)$ promediaron valores más elevados de Apego al Lugar (t[637] = 3.64, $p<0.001)$ e Identidad de Lugar $(t[744]=5.18, p<0.001)$ que los participantes que carecían de dicha propiedad (61,7\%) (Ver Figura 4). El tamaño de efecto correspondiente a estas diferencias es pequeño tanto para el caso de Apego al Lugar ( $d=0.26$; IC95\% [0.12 $0.41]$ ) como el de Identidad del Lugar ( $d=0.39$; IC95\% [0.25 - 0.54]).

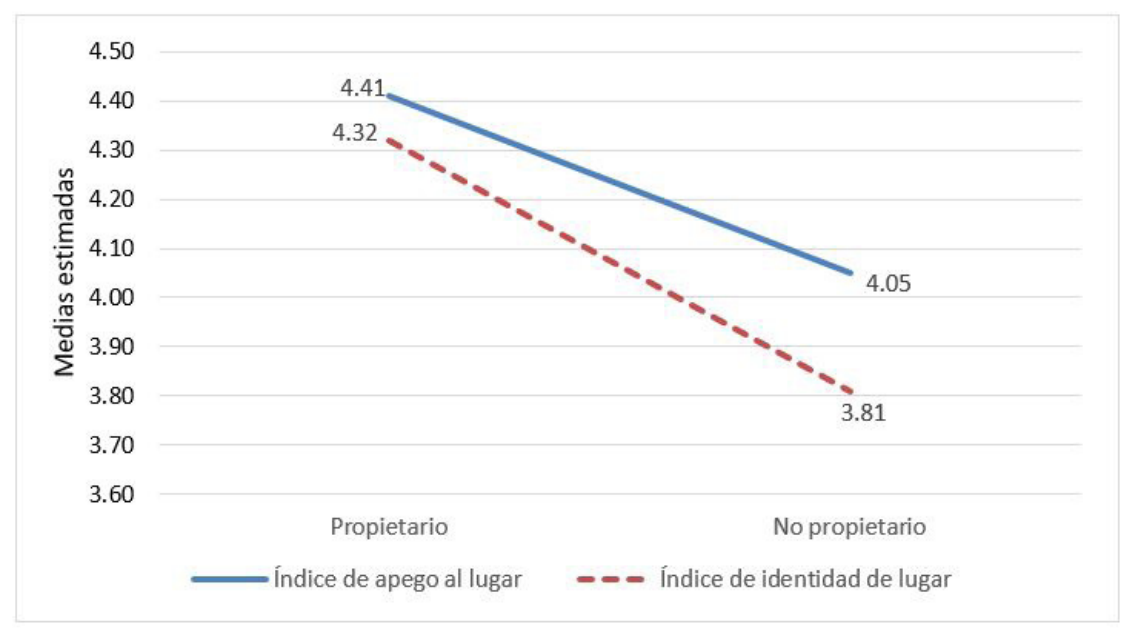

Figura 4. Medias de Apego al Lugar e Identidad de Lugar en función del régimen de propiedad.

Fuente: Datos recopilados por la Universidad de Valparaíso (Chile) y la Universidad Politécnica Salesiana (Ecuador). Elaboración propia.

A modo de síntesis, los resultados de las pruebas $t$ efectuadas muestran que factores como el tiempo de residencia, el tipo de barrio (patrimonial consolidado o patrimonial no consolidado) y el régimen de propiedad de la vivienda se relacionan con los niveles de Apego al Lugar e Identidad de Lugar que las personas experimentan. En este sentido, quienes tienen mayor tiempo de residencia, habitan zonas patrimoniales consolidadas y poseen su propia vivienda tienden a sentirse más apegados e identificados con el lugar en el que viven. Mientras la valoración de la condición patrimonial se relaciona únicamente con el Apego al Lugar, de modo que las personas con actitud favorable hacia el estatus patrimonial de su lugar de residencia, que corresponde al 89.2\% de los participantes, reportan niveles más elevados de Apego al Lugar. Finalmente, el carácter consolidado de los barrios patrimoniales constituye el único factor que produjo efectos moderados sobre el Apego al Lugar y la Identidad de Lugar, mientras que el resto de los factores con asociaciones significativas presentaron diferencias de medias de pequeña magnitud.

\section{Discusión}

Este estudio se propuso describir, analizar y comparar los niveles de Apego al Lugar e Identidad de Lugar de los habitantes de tres barrios patrimoniales de la ciudad de Valparaíso, Chile, y dos barrios patrimoniales de la ciudad de Quito, Ecuador. Los resultados descriptivos mostraron niveles moderados de Apego al Lugar e Identidad al Lugar en estos habitantes, no obstante, los de los barrios patrimoniales consolidados reportaron niveles superiores a los de los no consolidados. 
Pág 94

Las personas que viven en barrios patrimoniales consolidados, esto es, aquellos donde las dimensiones socio-urbanas del entorno, como efecto de la nominación patrimonial y las políticas de renovación urbana, se encuentran mayormente intervenidas y desarrolladas, muestran mayores niveles de Apego al Lugar e Identidad de Lugar que quienes habitan en barrios no consolidados.
Se planteó la hipótesis de que existen diferencias significativas en los niveles de Apego al Lugar e Identidad de Lugar según el tiempo de residencia, el tipo de barrio (patrimonial consolidado o patrimonial no consolidado), la ciudad, la valoración del estatus patrimonial y el régimen de propiedad de la vivienda. Con base en la evidencia empírica se puede sostener que la hipótesis de trabajo se verificó parcialmente.

En primer lugar, la relación entre el tiempo de residencia y las variables del estudio confirma, igual a lo reportado por Riger y Lavrakas (1981), Taylor, Gottfredson y Brower (1984), y Hernández, Hidalgo, Salazar-Laplace y Hess (2007), que mientras más años se reside en un lugar las personas presentan mayores niveles de Apego al Lugar e Identidad de Lugar.

En segundo lugar, las personas que viven en barrios patrimoniales consolidados, esto es, aquellos donde las dimensiones socio-urbanas del entorno, como efecto de la nominación patrimonial y las políticas de renovación urbana, se encuentran mayormente intervenidas y desarrolladas, muestran mayores niveles de Apego al Lugar e Identidad de Lugar que quienes habitan en barrios no consolidados. Este hallazgo puede ser explicado por la dimensión temporal (Hernández, Hidalgo, Salazar-Laplace, \& Hess, 2007) y por el tipo de interacciones sociales que se asocian a la condición patrimonial del barrio. Es decir, que en los barrios intervenidos se generan procesos de identificación simbólica de las personas respecto de aquellos aspectos físico-estéticos que han sido mejorados, a la vez que, son identificados como distintos por otros (turistas y no residentes); procesos que se van afianzando con el tiempo. Siguiendo a Rijnks y Strijker (2013), la identidad progresiva que va adquiriendo un lugar favorece la identificación de los individuos con el mismo, lo que finalmente se reflejaría en sus niveles de Identidad de Lugar. Esta cuestión abre interrogantes respecto a los procesos de vinculación socioespacial de los habitantes de barrios patrimoniales, dado que también se han evidenciado efectos negativos como resultado de las intervenciones realizadas en dichos lugares, relativos a procesos de gentrificación, aumento del turismo, exclusión social y fragilidad del sentido de comunidad, entre otros.

En tercer lugar, no se presentaron diferencias significativas en los niveles de Apego al Lugar e Identidad de Lugar según la ciudad. En los barrios de la ciudad de Valparaíso se obtuvieron puntajes mayores que en los barrios de la ciudad de Quito; situación que puede relacionarse con las características y dinámicas de trabajo que se desarrollaron en las intervenciones sociales y urbanas, y en el sentido de comunidad de los barrios de Quito. Al respecto, López (2014) y (Rodríguez, 2015) reportaron, en los barrios de "24 de mayo" y "La Ronda" en el centro histórico de Quito, respectivamente, los efectos excluyentes y de precarización de la participación ciudadana de algunos proyectos implementados en tales barrios, cuya orientación ha sido más comercial y turística (Hanley, 2008), que de promoción del patrimonio cultural. Diversos estudios han mostrado que los procesos de participación basados en compromisos de mejoras de los barrios inciden en el Apego al Lugar (Perkins \& Long, 2002; Manzo \& Perkin, 2006) y que la participación local está relacionada con el Apego al Lugar a través del sentido de comunidad (Vidal, Berroeta, Di Masso, Valera, \& Pero, 2013). McMillan y Chavis (1986) entienden el sentido de comunidad como el proceso a partir del cual los miembros de un entorno determinado generan vínculos de pertenencia con el mismo mediante el sentimiento de que importan los unos a los otros y la fe compartida de que las necesidades de cada uno serán atendidas mediante el compromiso de estar juntos. 
Pág 95

Las personas que evalúan positivamente el carácter patrimonial del barrio en el que viven presentan mayores niveles de Identidad de Lugar y de Apego al Lugar, que quienes presentan una valoración negativa.
En cuarto lugar, las personas que evalúan positivamente el carácter patrimonial del barrio en el que viven presentan mayores niveles de Identidad de Lugar y de Apego al Lugar, que quienes presentan una valoración negativa. No obstante, al establecer la diferencia de medias entre los grupos analizados, se constata que solo se presentaron diferencias estadísticamente significativas para el Apego de Lugar. Si bien no es posible realizar afirmaciones causales, por la naturaleza de las evidencias estadísticas reportadas, cabe suponer que la Identidad de Lugar no muestra una diferencia significativa porque es un proceso que, como señalan Hidalgo y Hernández (2001), Hernández et al. (2007) y Hernández, Martín, Ruiz e Hidalgo (2010), requiere más tiempo para afianzarse que el Apego al Lugar, en tanto, resulta de las interacciones permanentes con el entorno y con las personas del barrio. El carácter patrimonial del barrio ha sido parte reciente de la historia de estos barrios y podría entenderse como una dimensión valorativa que tiene más peso en el Apego al Lugar; por tanto, la Identidad del Lugar, certificada en su calidad de sitio patrimonio de la humanidad, no determinaría la identidad de lugar de sus habitantes.

En quinto lugar, al igual que los estudios clásicos de Riger y Lavrakas, (1981), Ringel y Finkelstein, (1991), y Taylor, Gottfredson y Brower (1984), los resultados del presente estudio muestran diferencias significativas en los niveles de Apego e Identidad de Lugar, según propiedad de la vivienda; de modo que quienes no son propietarios reportan menores niveles de Identidad de Lugar y Apego al Lugar, que los habitantes propietarios de sus viviendas.

Si bien no fue parte de los objetivos del estudio, cabe añadir la asociación encontrada entre las variables Apego e Identidad de Lugar, las cuales presentaron correlaciones elevadas, positivas y estadísticamente significativas tanto en Quito ( $r=0.74, p<$ $0.001)$ como en Valparaíso ( $r=0.82 ; p<0.001)$. En este sentido, los resultados de la presente investigación son coherentes con investigaciones previas (Lewicka, 2011, Vidal, Berroeta, Di Masso, Valera, \& Pero, 2013) que confirman que quienes se identifican con el barrio también se sienten apegados a él.

Los hallazgos de este estudio apoyan un conjunto de estudios psicoambientales que han venido mostrando la importancia de las variables: Apego al Lugar e Identidad de Lugar, para entender las relaciones y transacciones entre las personas y los entornos construidos.

\section{Conclusiones}

Los resultados de este estudio, cuya particularidad es que se desarrolló con habitantes de barrios patrimoniales, cualidad certificada por la UNESCO, confirman la necesidad de establecer una explicación teórica robusta que permita comprender la relación que se produce entre los lugares y las personas (Lewicka, 2011), y las diferencias entre Identidad de Lugar de las personas e Identidad de Lugar de los lugares. Para ello es necesario, en el ámbito de la Psicología Ambiental, transitar desde perspectivas psicológico-individuales a perspectivas semiótico-materiales (Berroeta, Pinto de Carvalho, Castillo-Sepúlveda, \& Opazo, 2020; Di Masso \& Dixon, 2015).

La promoción de lugares y su comercialización son prácticas fundamentales en los procesos de patrimonialización de las ciudades, que generan tanto dinámicas de inclusión como de exclusión social. Las identidades espaciales son creadas y recreadas por diversas personas interesadas; por tanto, es fundamental realizar 
estudios psicoambientales que den luces sobre los lazos emocionales e identitarios que establecen las personas residentes con estos lugares y que orienten soluciones prácticas para balancear sus necesidades y las de los promotores de ciudad.

\section{Referencias}

Ahlbrandt, R. (1984). Neighborhoods, people, and community. New York: Plenum Press.

Andrade, X. (2018). Perverso patrimonio: una mirada crítica desde la antropología. Persona y Sociedad, 32(1), 39-62. https://doi.org/10.11565/pys.v32i1.131

Berroeta, H. (2009). Simbolismo y acción colectiva en la configuración del espacio patrimonial urbano. En Pablo Andueza (Ed.) El patrimonio cultural como factor de desarrollo en Chile. pág. 59 -77. Universidad de Valparaíso

Boniato, M., Aiello, A., Perugini, M., \& Ercolano, A. P. (1999). Multidimensional perception of residential environment quality and neighbourhood attachment in the urban environment. Journal of Environmental Psychology, 19, 331-352. https:// doi.org/10.1006/jevp.1999.0138

Brown, G., \& Raymond, C. (2007). The relationship between place attachment and landscape values: Toward mapping place attachment. Applied geography, 27(2), 89-111. https://doi.org/10.1016/j.apgeog.2006.11.002

Brown, G., Brown, B.B., \& Perkins, D.D. (2004). New housing as neighborhood revitalization: Place attachment and confidence among residents. Environment \& Behavior, 36(6), 749-775. https://doi.org/10.1177/0013916503254823

Brown, B.B., Perkins, D.D., \& Brown, G. (2003). Place attachment in a revitalizing neighborhood: Individual and block levels of analysis. Journal of Environmental Psychology, 23, 259-271. https://doi.org/10.1016/S0272-4944(02)00117-2

Cabrera, V. (2008). Políticas de renovación en centros históricos de México. Centro-h, Revista de la Organización Latinoamericana y el Caribe de Centros Históricos, 1, 26-39. https://www.redalyc.org/articulo.oa?id=115112534003

Carrión, F. (2005). El centro histórico como proyecto y objeto de deseo. EURE (93), 89100. http://dx.doi.org/10.4067/S0250-71612005009300006

Casellas, A., \& Vergara-Constela, C. (2016). Políticas estatales y transformación urbana: ¿Hacia un proceso de gentrificación en Valparaíso Chile? EURE, 42(126), 123-144. http://dx.doi.org/10.4067/S0250-71612016000200006

Cuba, L., \& Hummon, D. (1993). A place to call home: identification with dwelling, community, region. The Sociological Quarterly, 34(1), 111-131.

Cuenya, B. (2011). Grandes proyectos y sus impactos en la centralidad urbana. Caderrnos Métropole 13(25), 185-212. https://revistas.pucsp.br/index.php/metropole/article/view/5987

Choay, F. (1992). Alegoría del Patrimonio. Barcelona: Gustavo Gili.

Dallago, L., Perkins, D. D., Santinello, M. Boyce, W., Molcho,, M., \& Morgan, A. (2009). Adolescent place attachment, social capital, and perceived safety: A comparison of 13 countries. American Journal of Community Psychology, 44(1-2), 148-160. https://doi.org/10.1007/s10464-009-9250-z

Di Masso, A., \& Dixon, J. (2015). More than words: place, discourse and the struggle over public space in Barcelona. Qualitative Research in Psychology, 12(1), 45-60

Fondo de Salvamento del Patrimonio Cultural, FONSAL. (2009). Recuperación Urbano Arquitectónica del Centro Histórico de Quito. Proyecto: Rehabilitación Urbano-Arquitectónica del eje de La Calle Morales "La Ronda" y su Área de Influencia. Décimo Foro de BIARRITZ. Recuperado de https://www.cmeal.org/documents/ 
renovacionquito ES.pdf

Galaz-Mandakovic, D. (2019). Las dos caras de la patrimonialización: memoria local y poética de la ausencia. Entorno, (67), 42-47. https://doi.org/10.5377/entorno. v0i67.7490

Giuliani, M. V. (2003). Theory of attachment and place attachment. En Bonnes, M., Lee, T., \& Bonito, M. (Eds.), Psychological theories for environmental issues, 137-170. Aldershot: Ashgate.

Guevara, T. (2011). ¿La ciudad para quién? Políticas habitacionales y renovación urbana en la boca. Cuaderno Urbano, 11(11), 81-99. http://dx.doi.org/10.30972/ crn.11111682

Gutman, M. (2001). Del monumento aislado a la multifuncionalidad. En F. Carrión (Ed.) Centros históricos de América Latina y el Caribe (pp. 95-106). Quito, Ecuador: UNESCO-FLACSO.

Hanley, L. (2008). Centros históricos: espacios de rehabilitación y disputa. Centro-h de la Organización Latinoamericana y del Caribe de Centros Históricos - OLACCHI, 1, 78-84. https://www.redalyc.org/articulo.oa?id=115112534007

Hernández, B., Hidalgo, M., Salazar-Laplace, M., \& Hess, S. (2007). Place Attachment and place identity in natives and non-natives. Journal of environmental psychology 27(4), 310-319. https://doi.org/10.1016/j.jenvp.2007.06.003

Hernández, B., Martín, A. M., Ruiz, C., \& Hidalgo, M. d. C. (2010). The role of place identity and place attachment in breaking environmental protection laws. Journal of Environmental Psychology, 30(3), 281-288. https://doi.org/10.1016/j.jenvp.2010.01.009

Hidalgo, M., \& Hernández, B. (2001). Place attachment: Conceptual and empirical questions. Journal of Environmental Psychology, 21(3), 273-281. https://doi. org/10.1006/jevp.2001.0221

Kingman, E. (2004). Patrimonio, políticas de la memoria e institucionalización de la cultura". ICONOS, Revista de Ciencias Sociales, 20, 26-34. https://doi. org/10.17141/iconos.20.2004.66

Kingman, E., \& Prats, L. (2008). El patrimonio, la construcción de las naciones y las políticas de exclusión. Diálogo sobre la noción de patrimonio. Centro-h de la Organización Latinoamericana y del Caribe de Centros Históricos - OLACCHI, 1, 87-97. https: / / www.redalyc.org/articulo.oa?id $=115112534008$

Lacarrieu, M. (2004). El patrimonio cultural inmaterial: un recurso político en el espacio de la cultura pública local. En VI Seminario sobre Patrimonio Cultural, llevado a cabo en Santiago, Chile. Recuperado de http://www.patrimoniocultural.gob.cl/ seminario 2004/pdf/capt 07 seminario.pdf

Lacarrieu, M., \& Laborde, S. (2018). Diálogos con la colonialidad: los límites del patrimonio en contextos de subalternidad. Persona y Sociedad, 32(1), 11-38. https:// doi.org/10.11565/pys.v32i1.130

Latiff, K., Imm, S., Abdul, Y., \& Kamal, N. (2020). Antecedent and outcome of place attachment in heritage sites. Journal of Applied Structural Equation Modeling, 4(1), 81-90. https://doi.org/10.47263/JASEM.4(1)06

Leiva, F., \& Díaz, P. (2020). Patrimonialización, desarrollo territorial y nuevos modelos de gobernanza. El caso del ramal ferroviario Talca-Constitución en la región del Maule, Chile. OPERA, 26, 37-53. https://doi.org/10.18601/16578651.n26.04

Lewicka, M. (2011). Place attachment: How far have we come in the last 40 years? Journal of Environmental Psychology, 31(3), 207-230. https://doi.org/10.1016/i. jenvp.2010.10.001

López, E. (2014). Incidencia de la participación ciudadana en las políticas de rehabilitación urbana: el caso de la Avenida 24 de Mayo, Quito - Ecuador (Tesis de título maestría) 
FLACSO, Ecuador. Recuperado de http://hdl.handle.net/10469/7486

Loureiro, S. M. C., \& Sarmento, E. M. (2018). Place attachment and tourist engagement of major visitor attractions in Lisbon. Tourism and Hospitality Research, 18(1), 110-127. https://doi.org/10.1177/1467358418761211

Manzo, L. C. (2003). Beyond house and haven: Towarda revisioning of emotional relationships with places. Journal of Environmental Psychology, 23(1), 47-61. https:// doi.org/10.1016/S0272-4944(02)00074-9

Manzo, L., \& Perkins, D. (2006). Finding common ground: The importance of place attachment to community participation and planning. Journal of Planning Literature, 20(4), 335-350. https://doi.org/10.1177/0885412205286160

Marasco, A., Buonincontri, P., \& Ramkissoon, H. (8-12 July 2018). Place attachment and sustainable behaviour in heritage tourism [Oral presentation]. International Association People-Environment Studies, Roma, Italy.

McMillan, D., \& Chavis, D. (1986). Sense of community: A definition and theory. Journal of Community Psychology, 14(1), 6-23.

Peng J., Strijker D., \& Wu, Q. (2020). Place Identity: How Far Have We Come in Exploring Its Meanings? Front. Psychol. 11, 294. https://doi.org/10.3389/fpsyg.2020.00294

Perkins, D., \& Long, D. (2002). Neighborhood sense of community and social capital: A multi-level analysis. En A. T. Fisher, C. C. Sonn \& B. J. Bishop (Eds.), Psychological sense of community: Research, applications, and implications (pp. 291-318). New York, NY: Kluwer Academic/Plenum Publishers. https://doi.org/10.1007/978-14615-0719-2 15

Pretty, G.H., Chipuer, H., \& Bramston, P. (2003). Sense of place amongst adolescents and adults in two rural Australian towns: The discriminating features of place attachment, sense of community and place dependence in relation to place identity. Journal of Environmental Psychology, 23(3), 273-287. https://doi. org/10.1016/S0272-4944(02)00079-8

Proshansky H. M., Fabian, A. K., \& Kaminof, R. (1983). Place-identity: Physical world socialization of the self. Journal of Environmental Psychology, 3(1), 57-83. https:// doi.org/10.1016/S0272-4944(83)80021-8

Rijnks, R. H., \& Strijker, D. (2013). Spatial effects on the image and identity of a rural area. J. Environ. Psychol. 36, 103-111. https://doi.org/10.1016/j.jenvp.2013.07.008

Riger, S., \& Lavrakas, P. (1981). Community ties: Patterns of attachment and social interaction in urban neighborhoods. American Journal of Community Psychology, 9(1), 55-66. https://doi.org/10.1007/BF00896360

Ringel, N.B., \& Finkelstein, J.C. (1991). Differentiating neighborhood satisfaction and neighborhood attachment among urban residents. Basic and Applied Social Pyschology, 12, 177-193. https://doi.org/10.1207/s15324834basp1202 4

Rodríguez, M. (2015). Renovación del barrio patrimonial La Ronda en Quito. Producción del espacio urbano, 2005-2013. Quito, Ecuador: Flacso.

Salcedo, R. (2007). La lucha por el espacio urbano. En O. Segovia (ED.) Espacios públicos y construcción social. Hacia un ejercicio de ciudadanía (pp. 69-77). Santiago, Chile: Ediciones SUR.

Scannell, L., \& Gifford, R. (2010a). Defining place attachment: A tripartite organizing framework. Journal of Environmental Psychology, 30(1), 1-10. https://doi. org/10.1016/j.jenvp.2009.09.006

Scannell, L., \& Gifford, R. (2010b). The relations between natural and civic place attachment and pro environmental behavior. Journal of Environmental Psychology, 30(3), 289-297. https://doi.org/10.1016/j.jenvp.2010.01.010

Suárez, C. (2010). Renovación urbana. ¿Una respuesta al pánico moral? Territorios 22. 111-124. https://revistas.urosario.edu.co/index.php/territorios/article/ 
Su, M. M., \& Wall, G. (2010). Place attachment and heritage tourism at the Great Wall. Journal of China Tourism Research, 6(4), 396-409. https://doi.org/10.1080/1938 $\underline{8160.2010 .527577}$

Taylor, R., Gottfredson, S., \& Brower, S. (1984). Neighborhood naming as an index of attachment to place. Population and Environment, 7(2),103-125. https://doi. org/10.1007/BF01254780

Tuan, Y. (1974). Topophilia: A study of environmental perception, attitudes and values. Englewood Cliffs (N.J.): Prentice Hall.

Valera, S. (1996). El significado del espacio urbano. Perspectivas teóricas y disciplinares. En S. Valera (Ed.) El significado social del espacio, estudio de la identidad social y los aspectos simbólicos del espacio urbano desde la psicología ambiental, (pp. 26-54). Barcelona, España: Cer Polis.

Vidal, T., Berroeta, H., Di Masso, A., Valera, S. \& Pero, M. (2013). Apego al lugar, identidad de lugar, sentido de comunidad y participación en un contexto de renovación urbana. Estudios de Psicología. Estudios de Psicología, 34(3), 275-286.

Vidal, T., Valera, S., \& Peró, M. (2010). Place attachment, place identity and residential mobility in undergraduate students. Psychology, 1(3), 353-369. https://doi. org/10.1174/217119710792774799

Williams, D. R., Patterson, M. E., Roggenbuck, J. W., \& Watson, A. E. (1992). Beyond the commodity metaphor: Examining emotional and symbolic attachment to place. Leisure Sciences, 14, 29-46. https://doi.org/10.1080/01490409209513155

Woosnam, K. Aleshinloye, M. Ribeiro, D. Stylidis, J. Jiang, J., \& Erul, E. (2018). Social determinants of place attachment at a World Heritage Site. Tourism Management, 67 (2018), pp. 139-146. https://doi.org/10.1016/j.tourman.2018.01.012 